\title{
Frontières
}

\section{Les conduites à risque sont-elles des conduites suicidaires ?}

\section{Joseph Lévy}

Volume 12, numéro 1, automne 1999

Suicides, générations et culture

URI : https://id.erudit.org/iderudit/1074506ar

DOI : https://doi.org/10.7202/1074506ar

Aller au sommaire du numéro

Éditeur(s)

Université du Québec à Montréal

ISSN

1180-3479 (imprimé)

1916-0976 (numérique)

Découvrir la revue

Citer cet article

Lévy, J. (1999). Les conduites à risque sont-elles des conduites suicidaires ? Frontières, 12(1), 39-42. https://doi.org/10.7202/1074506ar

\section{Résumé de l'article}

Les conduites extrêmes constituent, dans les sociétés contemporaines, des pratiques significatives. Leurs rapports avec la mort sont complexes et encore mal connus, les perspectives théoriques encore imprécises. Les recherches menées sur ce sujet suggèrent néanmoins qu'elles peuvent s'inscrire dans des dynamiques multiples : vivre pleinement sa vie, s'ancrer dans un sentiment d'invincibilité, constituer un équivalent suicidaire, s'inscrire dans des rituels ordaliques ou se situer dans une perspective directement suicidaire. Afin de mieux comprendre ces conduites, il est aussi important de tenir compte des différences sexuelles et des rapports de rivalité mimétique qui peuvent en moduler l'expression. 


\section{Résumé}

Les conduites extrêmes constituent, dans les sociétés contemporaines, des pratiques significatives. Leurs rapports avec la mort sont complexes et encore mal connus, les perspectives théoriques encore imprécises. Les recherches menées sur ce sujet suggèrent néanmoins qu'elles peuvent s'inscrire dans des dynamiques multiples: vivre pleinement sa vie, s'ancrer dans un sentiment d'invincibilité, constituer un équivalent suicidaire, s'inscrire dans des rituels ordaliques ou se situer dans une perspective directement suicidaire. Afin de mieux comprendre ces conduites, il est aussi important de tenir compte des différences sexuelles et des rapports de rivalité mimétique qui peuvent en moduler l'expression.

Mots-clés: suicide - conduites extrêmes rituels ordaliques - rivalité mimétique différences sexuelles

\begin{abstract}
Extreme behaviors constitute, in contemporary societies, revealing practices. Their connections to death are complex and less well known; theoretical models are even more inaccurate. Studies conducted on this subject suggest nevertheless that they fit into multiple dynamics: living one's life fully, settling into a feeling of invincibility, forming a suicidal equivalent, keeping with ordalic rituals or being placed in an inevitably suicidal outlook. To better understand these behaviors, one should take into account sexual differences and mimetic rivalry relations, which can vary the expression thereof.
\end{abstract}

Key words: suicide - extreme behaviors ordalic rituals- mimetic rivalry - sexual differences

\section{Les conduites à risque sont-elles des conduites suicidaires?}

\section{Joseph Lévy, \\ professeur au Département de sexologie de I'UQAM.}

Parler de comportements à risque fait maintenant partie du vocabulaire de nos contemporains. On fait alors référence à toute conduite qui augmente les probabilités, pour un individu qui les pratique, d'affecter son intégrité physique, sa santé physique et mentale, ce qui peut aller jusqu'à la mort directe ou, dans certains cas, différée.

Ces conduites à risque s'accompagnent d'états de conscience modifiés par la transformation des paramètres temporels, des modalités de l'action, de la sensibilité et des relations interpersonnelles ${ }^{1}$. Ces états de conscience peuvent prendre place dans le contexte de rituels bien définis, d'activités ludiques ou, au contraire, dans celui d'activités quotidiennes plus ou moins formalisées.

Les conduites à risque peuvent prendre plusieurs formes et nous retiendrons ici quelques catégories essentielles. Ainsi, on peut distinguer avec
P. Goëtze et C. Deneke ${ }^{2}$, les conduites à risque chroniques qui, à long terme, peuvent entraîner la mort, des conduites à risque simple ou répétitif qui incorporent la possibilité ici et maintenant de mourir.

Dans la première catégorie, on peut placer l'usage des produits licites (tabac et médicaments), des drogues illicites, des alcools et les conduites alimentaires problématiques (boulimie, anorexie nerveuse), tandis que dans la seconde, on retrouve essentiellement les activités liées aux sports extrêmes (conduite automobile, de hors-bord ou de motocyclette à grande vitesse, escalade, parachute, etc.) ou à des pratiques associées à des formes de roulette russe (volant lâché à grande vitesse, traversée de la voie automobile au rouge, duel automobile où deux voitures foncent l'une vers l'autre jusqu'à ce que l'un des deux conducteurs cède la route).

Ces conduites varient en fonction de l'âge, de l'origine ethnoculturelle, du milieu socio-économique et du sexe. 
Par exemple, des travaux menés en France $^{3}$ montrent des différences à la période de l'adolescence, selon le sexe. Les garçons abusent plus des drogues et de l'alcool, d'où des expériences d'ébriété plus fréquentes comparativement aux filles, qui consomment en plus grandes quantités des médicaments psychotropes et qui souffrent plus de troubles dans les conduites alimentaires. Les accidents liés à la conduite automobile sont plus fréquents chez les garçons 4 .

On peut aussi observer dans le recours aux conduites à risque, des configurations variées selon la fonction qu'occupent ces conduites au plan sociopsychologique. Ainsi, Le Breton ${ }^{5}$ oppose celles par excès d'intégration (i.e. pour augmenter les stimuli dans une société où dominent routine et sécurité) à celles par défaut d'intégration (i.e. liés à un manque, à une souffrance personnelle).

La perspective objective des conduites à risque n'est pas obligatoirement partagée par les acteurs impliqués. Ceux-ci, en particulier les adolescents, peuvent sous-évaluer les risques ou, même s'ils sont évalués correctement, ne pas tenir compte des conséquences qui en découlent. Selon F.

Zucker-Rouvillois 6 , les jeunes se caractérisent ainsi par une faible sensibilité aux dangers de la route et une relative indifférence aux dangers associés à la consommation excessive de tabac.

\section{LES CONDUITES À RISQUE, LE SUICIDE ET LES ACTES SUICIDAIRES}

Si les conduites à risque peuvent provoquer la mort accidentelle, interviennent-elles lorsqu'il y a suicide ou tentatives de suicidaire? Précisons d'abord ce que nous entendons ici par suicide, ce concept ayant donné naissance à de nombreux débats à partir desquels plusieurs distinctions ont été proposées. Ainsi, on considérera un suicide comme

tout acte, par commission ou omission, accompli par soi-même ou par d'autres, au moyen duquel un individu de façon autonome a l'intention et désire provoquer sa mort parce qu'il veut être mort ou veut mourir de la mort qu'il réalise ${ }^{7}$.

L'intention et le désir de mourir sont nécessaires pour qu'il y ait suicide. Cet acte se distinguerait de la «roulette cosmique» (où les individus abandonnent à une divinité ou au cosmos leur destinée, qui décidera de leur vie ou de leur mort) et de la posture suicidaire» (suicide gesturing) où l'acte posé peut ressembler à

LES CONDUITES À RISQUE PEUVENT AUSSI ÊTRE L'EXPRESSION

D'UN RAPPORT FAUSSÉ AVEC LA MORT,

EN PARTICULIER CHEZ LES ADOLESCENTS.

un suicide bien qu'il soit accompli avec l'intention de rester vivant.

Il n'existe pas, par ailleurs, de perspectives théoriques bien établies qui peuvent rendre compte des rapports entre les formes de suicide et les conduites à risque; on peut cependant dégager plusieurs cas de figure. Selon une première perspective, les conduites à risque sont l'expression d'une volonté de vivre pleinement sa vie en se plaçant dans des conditions limites qui permettent de faire l'expérience de sa plénitu$\mathrm{de}^{8}$. Défaire son ennui, «s'éclater», selon la terminologie à la mode seraient à la base de telles conduites. On pourrait comprendre, par exemple, l'engouement pour les pratiques sportives extrêmes à partir d'une telle perspective. Certains de ces sports feraient partie de l'ilynx, une des catégories ludiques définies par R. Caillois où «il s'agit d'accéder à une sorte de spasme, de transe ou d'étourdissement qui anéantit la réalité avec une souveraine brusquerie ${ }^{9}{ }$. Comme le souligne J. Baechler à ce sujet:

les courses en auto, l'alpinisme, les cascades, la voltige sont des jeux vertiges qui tirent leur pouvoir d'attraction du danger qu'ils recèlent et de la victoire remportée sur lui et la peur. L'on sait que la mentalité typique de ceux qui pratiquent ces jeux est caractérisée par le refus systématique d'envisager la possibilité même d'un accident et par l'effort

constant pour atteindre à un automatisme parfait des gestes ${ }^{10}$.

Cet automatisme des gestes contribue à l'expérience euphorique du «flow» 11 , où tout semble s'intégrer dans un ensemble harmonieux: être, environnement et gestes. À titre d'exemple, une étude sur l'alpinisme ${ }^{12}$ montre que pour les pratiquants de ce sport, plusieurs éléments entrent en jeu: le défi mental et le défi physique extrêmes, le risque objectif et subjectif de mourir, la maîtrise du danger grâce à des habiletés spécifiques et la présence d'une passion. Ce type de sports permettrait de réduire les problèmes d'identité liés en particulier à des sentiments d'aliénation et de désintégration. La stimulation procurée par ces sports s'accompagne d'une évaluation soigneuse du matériel utilisé et l'occurrence d'un accident mortel ne peut donc être attribuable à une intentionnalité de suicide strictement définie. Néanmoins, cette position théorique ne fait pas l'unanimité. Certains chercheurs voient au contraire dans la quête de tels risques la présence d'une tendance suicidaire qui parcourrait l'ensemble du champ social. Selon P. Baudry ${ }^{13}$ «la promotion d'un appétit de vivre [...] tend à ne plus pouvoir se satisfaire que dans le risque encouru que ça casse». La logique sportive poussée, en particulier chez ceux qui sont engagés dans le sport amateur et dans le sport professionnel où le culte de la performance est poussé à un point tel que l'on assiste à la prolifération de postures suicidaires sinon même à des suicides.

Les conduites à risque peuvent aussi être l'expression d'un rapport faussé avec la mort, en particulier chez les adolescents. Comme le souligne É. ZuckerRouvillois ${ }^{14}$, la mort à cet âge paraît à la fois peu réelle, éloignée et réversible, ce qui peut contribuer à un sentiment d'invincibilité et empêcher une évaluation réaliste des conséquences des actes. Si la mort survient, elle ne peut être définie comme une forme de suicide.

Ces comportements peuvent aussi être envisagés comme «équivalent suicidaire», selon l'expression de A. Tursz ${ }^{15}$ et s'inscriraient dans des procédures de type ordalique "où l'on risque sa vie pour s'éprouver soi-même ou solliciter le jugement des dieux» 16 , l'enjeu étant de prouver son innocence, son bon droit, la (sa) vérité ou démontrer que la vie mérite d'être vécue, selon les catégories définies par cet auteur. Il serait cependant nécessaire de nuancer cette catégorisation en y intégrant, par exemple, la quête de l'authentification de son identité. C'est le cas, par exemple, des conduites visant à assurer à soi-même et au regard de l'autre son identité sexuelle.

Les conduites hypermasculines incluent la fascination pour le danger, un scénario défensif contre les affects de honte que l'on retrouverait chez les individus machistes ${ }^{17}$. La validation de la masculinité passe alors par l'expérimen- 
tation du danger qui permet de s'assurer que l'on est un vrai homme, d'où le recours à des conduites à risque qui peuvent s'inscrire dans un contexte interpersonnel qui pourrait sous-tendre de tels comportements amplifiés par une rivalité mimétique ${ }^{18}$. Cette rivalité pousserait les individus à un surenchérissement des conduites ordaliques. Les rituels ordaliques, avec la sécularisation de la société, exigent une prise de risques qui consiste soit à accepter les probabilités liées aux conduites à risque soit à agir sur elles ${ }^{19}$. Franchir les feux rouges, jouer à la roulette russe «cosmique», par exemple, feraient partie de cette catégorie. Il n'est donc pas sûr dans le cas des conduites ordaliques, que la mort soit véritablement recherchée comme c'est le cas dans le suicide planifié et volontaire.

Si de telles conduites peuvent servir à tester le courage personnel, certains chercheurs les considèrent comme une forte pulsion parasuicidaire subconsciente $^{20}$. On peut rapprocher ces conduites de celles que l'on retrouve dans certaines configurations de personnalité dites perverses, selon le courant psychanalytique. Ainsi, selon Y. Prigent,

les prises de risques, aux limites

de la démarche suicidaire, ne représentent pas une rencontre malencontreuse avec la malchance, ou une audace calculée et ponctuelle appliquée par une nécessité d'occasion, mais bien un modèle de vie affective où, pour dire en un raccourci, les limites de la loi [...] sont remplacées par les limites de la mort.

Cette fonction de la mort partenaire dans la vie du pervers explique qu'il s' en débarrasse rarement d'un coup mais qu'il entretient avec elle une sorte de flirt dont l'issue est souvent aussi radicale qu'un suicide au sens habituel du terme ${ }^{21}$.

Dans un dernier cas de figure, les idées suicidaires, les tentatives de suicide, les suicides planifiés et réussis se trouvent souvent associés ou sous-tendus par des pratiques à risque, en particulier l'usage des drogues et de l'alcool chez les jeunes adultes ${ }^{22}$. Une étude menée en Norvège ${ }^{23}$ montre ainsi une covariation entre les tentatives de suicide et des épisodes de surdose de drogues. Une même association se retrouve parmi un groupe de toxicomanes américains chez qui l'on constate des corrélations significatives entre les pensées suicidaires et les tentatives de suicide de même qu'entre les pensées suicidaires, les surdoses non suicidaires et les arrestations pour conduite automobile sous effet des drogues. Les tentatives de suicide sont aussi au moins 12 fois plus importantes que dans la population en général ${ }^{24}$. Selon une autre étude menée auprès de jeunes toxicomanes, les idées suicidaires et les tentatives de suicide étaient rapportées par une majorité significative de répondants, en particulier les femmes ${ }^{25}$.
Aux États-Unis, des études épidémiologiques ont montré un niveau d'alcoolémie qui dépassait les taux légalement acceptables: sur un échantillon de 100000 morts, $5 \%$ des personnes décédées suite à un suicide avaient un taux élevé de substances alcooliques dans le sang ${ }^{26}$, association que l'on retrouvait aussi chez des suicidés qui avaient utilisé des armes à feu pour commettre leur geste 27 . Une autre étude montre qu'entre $20 \%$ et $35 \%$ des suicidés avaient eu une histoire d'abus d'alcool ou avaient ingéré de tels produits un peu avant de se suicider ${ }^{28}$.

En France, tant des études anciennes que récentes semblent indiquer une même tendance. Ainsi selon Baechler ${ }^{29}$, des données statistiques montrent que les sujets drogués ont des postures suicidaires bien affirmées. Ainsi, 115 actes suicidaires - dont 4 réussis - avaient été commis par 35 sujets drogués. Plus récemment $\mathrm{M}$. Choquet et $\mathrm{H}$. Menke ${ }^{30}$ ont établi la présence chez les adolescents de relations entre les idées suicidaires et la présence de conduites à risque (tabac, alcool, drogues illicites, médicaments), tendances qui se retrouvent aussi aux États-Unis. Parmi les jeunes, l'abus de drogues et d'alcool se retrouvait chez $70 \%$ des suicidés ${ }^{31}$.

Plusieurs hypothèses ont été avancées pour expliquer ces associations. La consommation de ces substances, indépendamment ou en concomitance, peut ainsi jouer directement sur les mécanismes neuropsychologiques en réduisant les inhibitions et les mécanismes de

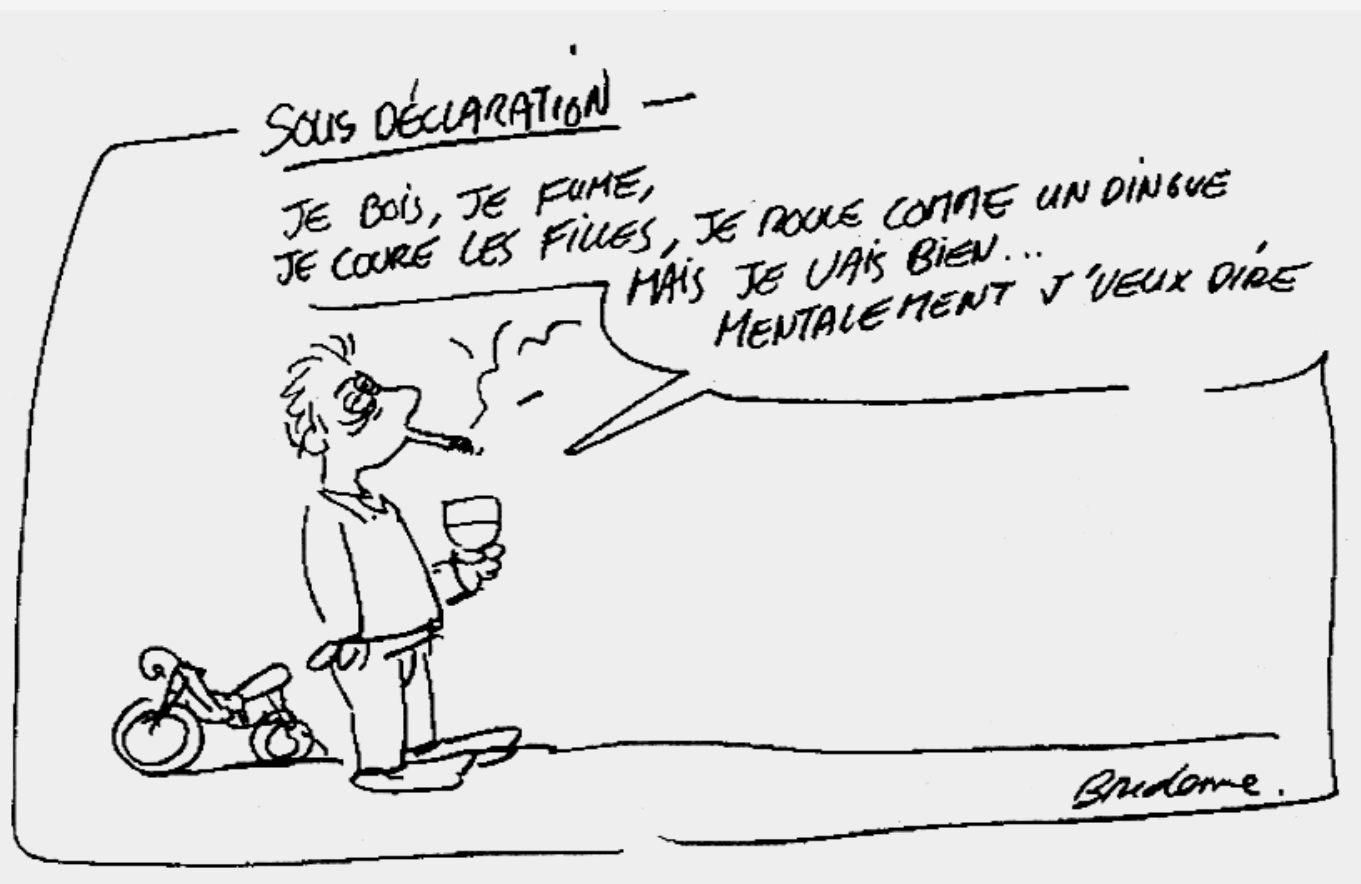


défense, ce qui affecte le jugement d'un individu ayant des idées suicidaires, d'où le passage à l'acte. Il semble cependant que ces substances soient associées à des suicides planifiés plutôt qu'impulsifs. Ces substances peuvent aussi avoir un effet indirect sur les conduites suicidaires en amplifiant la gravité des troubles mentaux sous-jacents à des actes suicidaires, comme la dépression ou d'autres troubles psychiatriques qui peuvent contribuer au suicide ${ }^{32}$.

\section{CONCLUSIONS}

Ce tour d'horizon illustre comment les comportements à risque entretiennent des rapports complexes avec la mort, les conduites suicidaires et le suicide. Ces rapports sont encore mal cernés et les modèles théoriques, surtout holistiques, qui peuvent les expliquer sont encore à développer. Tout un programme de recherche dans ce domaine est donc à réaliser et ce, à la fois dans une perspective socioculturelle et ontogénique. Il sera ainsi possible de mieux comprendre les motivations qui interviennent dans la prise de risques, les représentations de la mort qui les accompagnent et la dynamique de la vie fantasmatique qui peut intervenir dans les idéations suicidaires, le passage à l'acte, de même que le choix des moyens. Les rapports sociaux qui les sous-tendent demandent aussi à être mieux saisis si l'on veut rendre compte d'un phénomène qui tend à prendre une importance grandissante dans le contexte social contemporain.

\section{Notes}

1 H. COHEN et J.J. LÉVY, Les états de conscience modifiés, Montréal, Éditions du Méridien, 1988.

2 P. GÖTZE et C. DENEKE, «Risk Taking Behavior Versus Parasuicide in Group Behavior among Adolescents», dans Suicidal Behavior, the State of the Art, K. BÖHME et al. (dir. publ.), Regensburg, S. Roderer, 1993, p. 469-473.

3 Voir par exemple M. CHOQUET et R. BOYER, «Les différences entre les garçons et les filles à travers des enquêtes sociologiques et épidémiologiques», dans Adolescentes, adolescents, psychopathologie différentielle, A. BRACONNIER (dir. publ. ) avec la collaboration de C. CHILAND et al., Paris, Bayard, 1995, p. 43-57.

É. FOMBONNE, «Psychopathologie de garçons et des filles: données épidémiologiques et transformations de l'adolescence», ibid., p. 24-42.

I. GASQUET et M. CHOQUET, «Spécificité du comportement suicidaire des garçons à l'adolescence: implications thérapeutiques», ibid., p. 81-91.

4 R. POMARÈDE, «Morbidité hospitalière liée aux conduites de risque à l'adolescence», dans Adolescence et risque, A. TURSZ, Y. SOUTEYRAND et R. SALMI (dir. publ.), Paris, Éditions Syros, 1993, p. 103-110.

5 D. LE BRETON, «Les activités à risques: éthique du jeu avec la mort», Cahier de recherche éthique, no 19, 1996, p. 165-178.

6 É. ZUCKER-ROUVILLOIS, «La mortalité liée au risque à l'adolescence», dans Adolescence et risque, A. TURSZ, Y. SOUTEYRAND et R. SALMI (dir. publ.), Paris, Éditions Syros, 1993, p. 89-102.

7 G.J. FAIRBAN, "Contemplating Suicide. The language and Ethics of Self Harm», Londres et New York, Routledge, 1995, p. 84.

8 É. ZUCKER-ROUVILLOIS, ibid.

9 R. CAILlOIS, Les jeux et les hommes, Paris, Gallimard, 1958, p. 68.

$10 \mathrm{~J}$. BAECHLER, Les suicides, Paris, Calmann-Lévy, 1955, p. 259.

$11 \mathrm{M}$. CSIKSZENTMIHALYI, "Flow, the Psychology of Optimal Experience, New York, Harper and Row, 1990.

12 U. AUFMUTH, cité dans P. GÖTZE et C. DENEKE, «Risk Taking Behavior Versus Parasuicide in Group Behavior among Adolescents» dans Suicidal Behavior, the State of the Art, K. BÖHME et al. (dir. publ.), 1993, p. 469-473,

13 P. BAUDRY, Le corps extrême. Approche sociologique des conduites à risque, Paris, L'Harmattan, 1991, p. 55.

14 É. ZUCKER-ROUVILLOIS, ibid.

15 A. TURSZ, «Des diverses approches du risque à l'adolescence, dans Adolescence et risque, A. TURSZ, Y. SOUTEYRAND et R. SALMI (dir. publ.), Paris, Éditions Syros, p. 135-152.

16 J. BAECHLER, ibid., p. 238. D. LE BRETON, Passion du risque, Paris, Métailié, 1991.

17 D. MOSHER et S. TOMKINS, «Scripting the Macho Man», Journal of Research in Personnality, no 18, 1988, p. 150-163.
18 R. GIRARD La violence et le sacré, Paris, Hachette, 1998.

19 Voir J. BAECHLER, ibid, p. 240 et suivantes pour une discussion de cette question.

20 P. GÖTZE et C. DENEKE, ibid.

21 Y. PRIGENT, La souffrance suicidaire. Essai sur le mal insupportable, Paris, Desclée de Brouwer, 1994, p. 84.

22 F.E. CRUMLEY, «Substance Abuse and Adolescent Suicidal Behavior», Journal of American Medical Association, vol. 263, no 22, 13 juin 1990, p. 3051-3061.

A. MINO, A. BOUSQUET et B. BROERS, «Substance Abuse and Drug-Related Death, Suicidal Ideation, and Suicide: A Review», Crisis, vol. 20, no 1, 1999, p. 2835

23 I. ROSSOW et G. LAURITZEN, "Balancing on The Edge of Death: Suicide Attempts and Life-Threatening Overdoses among Drug Addicts», Addiction, vol. 94 , no 2, février 1999, p. 209-219.

24 S. SAXON, E. KUNCEL et S. ALDRICH, «Drug Abuse and Suicide», American Journal of Drug and Alcohol Abuse, vol. 5, no 4, 1978, p. 485-495.

25 E.Y. DEYKIN et S.L. BUKA, «Suicidal Ideation and Attempts among Chemically Dependent Adolescents, American Journal Of Public Health, vol. 84, no 4, avril 1994, p. 634-639.

26 Eighth Special Report to the U.S. Congress on Alcohol and Health, septembre 1993.

27 Seventh Special Report to the U.S. Congress on Alcohol and Health, janvier 1990.

28 Seventh Special Report, ibid.

29 J. BAECHLER, ibid.

30 M. CHOQUET et H. MENKE, «Suicidal Thoughts During Early Adolescence, Prevalence, Associated Troubles and HelSeeking Behavior», Acta Psychiatrica Scandinavia, no 81, 1989, p. 170-177.

31 U.S. DEPARTMENT OF EDUCATION, Youth \& Alcohol: Selected Reports to the Surgeon General, 1993.

32 NATIONAL COMMITTEE FOR INJURY PREVENTION AND CONTROL, Injury Prevention: Meeting the Challenge, document officiel, Gouvernement des États-Unis, 1993. 